\title{
Clinical outcomes and nephrotoxicity of colistin loading dose for treatment of extensively drug-resistant Acinetobacter baumannii in cancer patients
}

\author{
This article was published in the following Dove Press journal: \\ Infection and Drug Resistance \\ 7 September 2017 \\ Number of times this article has been viewed
}

\author{
Wasan Katip' \\ Suriyon Uitrakul ${ }^{2}$ \\ Peninnah Oberdorfer ${ }^{3}$ \\ 'Department of Pharmaceutical \\ Care, Faculty of Pharmacy, Chiang \\ Mai University, Chiang Mai, Thailand; \\ ${ }^{2}$ Northern Institute for Cancer \\ Research, Newcastle University, \\ Newcastle upon Tyne, UK; ${ }^{3}$ Division \\ of Infectious Diseases, Department of \\ Pediatrics, Faculty of Medicine, Chiang \\ Mai University, Chiang Mai, Thailand
}

Correspondence: Wasan Katip Department of Pharmaceutical Care, Faculty of Pharmacy, Chiang Mai University, Chiang Mai 50200, Thailand

Tel +6653944334

Fax +665322274

Email wasankatip@gmail.com
Background: Colistin is a last-line defense therapy against extensively drug-resistant Acinetobacter baumannii (XDR-AB). Despite a loading dose of colistin being applied in many clinical practices, studies evaluating the effect of the loading dose of colistin in cancer patients remain limited.

Patients and methods: A retrospective cohort study of cancer patients who received either a loading or non-loading dose of colistin for treatment of XDR-AB was conducted. For each group, the clinical response, bacteriological eradication and serum creatinine were recorded. Logistic regression was applied to evaluate the effects of therapy on each of the three aforementioned outcomes.

Results: One hundred and two patients diagnosed with XDR-AB infections between January 2012 and December 2015 were recruited. Only 75 patients were given a loading dose of colistin. There was no significant clinical and microbiological response in patients in the loading dose group or patients in the non-loading dose group. However, 38 (50.67\%) patients in the loading dose group and $6(22.22 \%)$ patients in the non-loading dose group developed nephrotoxicity according to the RIFLE criteria $(p=0.013)$. Multivariate logistic regression analysis showed that independent predictors of clinical response were Charlson score $\geq 4$ and duration of colistin treatment $\geq 10$ days. Septic shock correlated with both poor clinical and microbiological response. Independent predictors for nephrotoxicity were loading dose colistin and patient's age $\geq 60$ years. Conclusion: Administration of colistin loading dose did not significantly increase clinical response, microbiological response or mortality rate compared to non-loading dose in cancer patients with XDR-AB-related infections. However, nephrotoxicity was significantly higher when patients received loading dose colistin.

Keywords: loading dose, colistin, cancer patients, extensively drug-resistant Acinetobacter baumannii, nephrotoxicity, clinical outcome

\section{Introduction}

Acinetobacter baumannii is one of serious nosocomial pathogens worldwide, particularly among severely ill patients including cancer patients and those receiving hematopoietic stem cell transplantation. ${ }^{1} A$. baumannii infections can be fatal in patients with suboptimal immune defenses, especially cancer patients. ${ }^{1-3}$ Patients with solid tumors who are infected with $A$. baumannii have the mortality rate of $14.5 \% .{ }^{4}$ For patients with both hematologic malignancies and $A$. baumannii infections, the mortality rate increased to $83 \% .^{5}$

Colistimethate sodium (CMS) was shown to be highly effective for treating extensively drug-resistant $A$. baumannii (XDR-AB) infections that were usually susceptible 
only to colistin. ${ }^{6}$ Pharmacokinetic modeling demonstrated that patients with $A$. baumannii infections who received a loading dose colistin had drug concentration within therapeutic range. Despite a loading dose of colistin was applied in many clinical practices, studies evaluating loading dose colistin in cancer patients remain limited. Furthermore, only a few studies described the outcomes of treating XDR-AB infections with colistin in cancer patients and they were all limited by small sample sizes. ${ }^{7-9}$

The primary objectives of this study were to determine the clinical response, microbiological response, and nephrotoxicity of administering a loading dose of colistin for treating XDR-AB-related infections in cancer patients. The secondary outcome was the overall mortality rate of cancer patients infected with XDR-AB.

\section{Patients and methods}

This retrospective cohort study was performed in cancer patients infected with $A$. baumannii who were admitted to Chiang Mai University Hospital, Chiang Mai, Thailand from January 2012 to December 2015.

The Ethics Committee on Human Research of the Faculty of Medicine, Chiang Mai University approved this study with a waiver of informed consent for retrospective data collection under the condition of anonymously stored data collected. We selected cancer patients with XDR-AB infections from medical chart records and the microbiology laboratory database. Infections were defined according to the Center for Disease Control and Prevention criteria. ${ }^{10}$ Patients were included if they were 18 years or older, received colistin for more than 48 hours to treat the documented XDR-AB infection and received only one course of colistin treatment. Patients were excluded if they had other types of gram-negative infections or received hemodialysis or renal replacement therapy. Baseline characteristics included age, sex, intensive care unit (ICU) admission during infection, Charlson score and serum creatinine. The recruited cancer patients with XDRAB infections were divided into two groups. The loading dose group included patients who received a loading dose of colistin ( 9 million IU of CMS; $300 \mathrm{mg}$ colistin base activity [CBA]) once at the start of treatment course and then regular dose (4.5 MIU; $150 \mathrm{mg} \mathrm{CBA}$ ) infused for 1 hour in every 12 hours. Patients in the other group received regular dose (150 mg CBA) every 12 hours since the start of treatment. Colistin dose was also adjusted in patients with renal impairment. In patients with moderate-to-severe renal impairment (creatinine clearance rate $<50 \mathrm{~mL} / \mathrm{min}$ ), the dose and dosing interval were adjusted according to Cockcroft and Gault creatinine clearance estimates: maintenance dose of $150 \mathrm{mg}$ CBA every 24 hours for creatinine clearance rate of $20-50 \mathrm{~mL} / \mathrm{minor}$ $150 \mathrm{mg}$ CBA every 48 hours for creatinine clearance rate of $<20 \mathrm{~mL} / \mathrm{min}$ was administered. The duration of colistin for each of patients depends on type of infection.

Antimicrobial susceptibility tests were performed according to the guidelines of the Clinical and Laboratory Standards Institute (CLSI) (formerly the National Committee for Clinical Laboratory Standards); E-test was used to determine susceptibility of $A$. baumannii. ${ }^{11}$ XDR-AB was defined as resistance to penicillins, cephalosporins, fluoroquinolones, aminoglycosides and carbapenems (colistin sensitive only). ${ }^{2,3}$

\section{Outcome assessment}

The clinical response of colistin was assessed by resolution or partial resolution of fever, leukocytosis and local signs and symptoms of XDR-AB-related infections at the end of treatment. Clinical failure was defined as failure to meet all criteria for clinical response. Microbiological response was defined as obtaining two consecutive negative XDR-AB cultures from the site of infection after the initial positive culture, whereas microbiologic failure was defined as persistence of the original causative organism in the subsequent specimen cultures. Nephrotoxicity was counted if patients developed any grades of renal failure based on RIFLE criteria. The RIFLE criteria contain three grades of acute renal failure (risk, injury and failure) and two long-term outcomes (loss and end-stage renal disease). ${ }^{12}$

\section{Statistical analysis}

Statistical analysis was performed using Stata software, version 14 (Stata-Corp, College Station, TX, USA). All variables were compared between the loading and non-loading dose groups. Mean with standard deviation and median with interquartile range were used for continuous data, while frequency and percentages were used to describe nominal data. Fisher's exact test was used to compare categorical variables. Continuous variables were compared by independent $t$-tests if they had normal distribution and Wilcoxon rank sum tests if the data had non-normal distribution. All statistical tests were considered two sided, and statistical significance was defined as $p \leq 0.05$.

Logistic regression analysis was used to determine the effects of colistin on three primary outcomes: clinical response, microbiological response and nephrotoxicity. All variables with $p<0.25$ by univariate analysis were further investigated by multivariate logistic regression analysis. In addition, all variables that demonstrated a trend toward 
association with outcomes were forced into the model at the discretion of the investigators, and statistical significance was set at $p \leq 0.05$.

\section{Results}

During the study period, 102 patients met the inclusion criteria; 75 had been treated with a loading dose of colistin and 27 had been treated with non-loading dose of colistin. Most patients were females (loading dose group, 62\%; non-loading dose group, $59 \% ; p=1.000$ ) (Table 1 ). The mean age in the loading dose group $(59.89 \pm 14.46)$ was not significantly different from the non-loading dose group $(65.18 \pm 11.81)$ $(p=0.091)$.

Type of patients' malignant tumors was similar between the loading dose and non-loading dose groups. Other baseline characteristics such as ICU status, septic shock incidence, total CMS dose and duration were similar between the two groups.

Table I Demographic and clinical characteristics of patients in the loading dose and non-loading dose groups

\begin{tabular}{|c|c|c|c|}
\hline Characteristic & $\begin{array}{l}\text { Loading dose } \\
(n=75)\end{array}$ & $\begin{array}{l}\text { Non-loading dose } \\
(n=27)\end{array}$ & $p$-value \\
\hline \multicolumn{4}{|l|}{ Sex (no. [\%] of patients) } \\
\hline Male & $29(38)$ & II (4I) & 1.000 \\
\hline Female & $46(62)$ & $16(59)$ & \\
\hline Age (years), mean $\pm S D$ & $59.89 \pm 14.46$ & $65.18 \pm 11.81$ & 0.091 \\
\hline Type of malignancy, $n(\%)$ & & & $0.74 I$ \\
\hline Solid & $66(88)$ & $23(85)$ & \\
\hline - Lung cancer & $17(22.67)$ & $5(18.52)$ & \\
\hline - Brain cancer & $6(8.00)$ & $2(7.4 I)$ & \\
\hline - Liver and bile duct cancer & $9(12.00)$ & $5(18.52)$ & \\
\hline - Urogenital cancer & $6(8.00)$ & $4(14.8 I)$ & \\
\hline - Colon cancer & $8(10.67)$ & $2(7.4 I)$ & \\
\hline - Bone cancer & $5(6.67)$ & $2(7.4 I)$ & \\
\hline - Head and neck cancer & $8(10.67)$ & I (3.70) & \\
\hline - Gynecologic cancer & $7(9.33)$ & $2(7.4 I)$ & \\
\hline Hematologic & $9(12)$ & $4(15)$ & \\
\hline - Lymphoma & $4(5.33)$ & $4(15)$ & \\
\hline - Leukemia & $5(6.67)$ & $0(0)$ & \\
\hline ICU status, n (\%) & $4 \mid(54.67)$ & $18(66.67)$ & 0.365 \\
\hline Septic shock, n (\%) & $39(52.00)$ & $19(70.37)$ & 0.117 \\
\hline Charlson score, mean \pm SD & $4.10 \pm 2.34$ & $4.44 \pm 2.45$ & 0.527 \\
\hline Baseline SCr, mg/dl, median (IQR) & $0.7(0.5-1.1)$ & $0.8(0.6-3)$ & 0.036 \\
\hline $\mathrm{SCr}$ increase from baseline, median (IQR) & $0.8(0.2-1.7)$ & $0.1(0-1.6)$ & 0.031 \\
\hline Baseline GFR, ml/min, median (IQR) & $100.09(60.1-117.8)$ & $75.2(2 \mid .4 I-110.9)$ & 0.019 \\
\hline Lowest GFR after colistin treatment, $\mathrm{ml} / \mathrm{min}$, median (IQR) & $34.4(16-88.9)$ & $28.13(11.6-79.6)$ & 0.453 \\
\hline Total CMS dose, g, median (min-max) & $2.25(4.95-10.35)$ & $1.95(0.3-7.5)$ & 0.2984 \\
\hline \multicolumn{4}{|l|}{ Type of nephrotoxic medications, ${ }^{*}$ n (\%) } \\
\hline Aminoglycosides & $5(6.76)$ & $2(7.4 I)$ & 1.000 \\
\hline Diuretics & $54(72)$ & $21(78)$ & 0.621 \\
\hline Amphotericin B & $6(8)$ & I (3.7) & 0.672 \\
\hline Vasopressor & $43(58.11)$ & $19(70.37)$ & 0.356 \\
\hline Vancomycin & $40(53.33)$ & $15(55.56)$ & 1.000 \\
\hline Duration of IV colistin (days), mean \pm SD & $9.56 \pm 5.18$ & $1 \mathrm{I} .14 \pm 5.90$ & 0.192 \\
\hline Length of hospital stay, days, mean \pm SD & $43.93 \pm 32.26$ & $44.29 \pm 28.55$ & 0.959 \\
\hline Source of infection & & & 0.318 \\
\hline Pneumonia & $53(70.67)$ & $16(59.26)$ & \\
\hline Bacteremia & $5(6.67)$ & I (3.70) & \\
\hline UTI & $12(16.00)$ & $5(18.52)$ & \\
\hline Other & $5(6.67)$ & $5(18.52)$ & \\
\hline Colistin MICs, median (min-max) & $0.25(0.094-1.5)$ & $0.25(0.064-1.5)$ & 0.898 \\
\hline
\end{tabular}

Notes: *One patient can have $>$ I drugs. Other represents inter costal drainage, pus from wound.

Abbreviations: SD, standard deviation; ICU, intensive care unit; SCr, serum creatinine; IQR, interquartile range; GFR, glomerular filtration rate; MIC, minimum inhibitory concentration; CMS, colistimethate sodium; IV, intravenous; UTI, urinary tract infection 


\section{Outcomes}

Only nephrotoxicity was significantly higher in patients in the loading dose group than those in the non-loading dose group. Other outcomes such as clinical response, microbiological response and overall mortality did not show significant difference between the two groups, although the clinical and microbiological response trended in favor of the loading dose group (Table 2).

\section{Univariate and multivariate logistic regression analysis of the outcomes}

Univariate analysis and multivariate logistic regression analysis (Table 3) showed that patients treated with colistin for more than 10 days demonstrated good clinical response, while Charlson score $\geq 4$ was related to poor clinical response in patients with XDR-AB infection. Moreover, septic shock was related to poor clinical and microbiological responses (Table 3). Finally, loading dose colistin was associated with nephrotoxicity.

Table 2 Overall outcomes and toxicity in loading dose and nonloading dose groups

\begin{tabular}{llll}
\hline Outcome & $\begin{array}{l}\text { Loading dose } \\
(\mathbf{n}=\mathbf{7 5})\end{array}$ & $\begin{array}{l}\text { Non-loading dose } \\
(\mathbf{n}=\mathbf{2 7})\end{array}$ & p-value \\
\hline Clinical response & $40(53.33)$ & $13(48.15)$ & 0.661 \\
$\begin{array}{l}\text { Microbiological } \\
\text { response }\end{array}$ & $55(73.33)$ & $15(55.56)$ & 0.097 \\
Nephrotoxicity & $\mathbf{3 8 ( 5 0 . 6 7 )}$ & $\mathbf{6 ( 2 2 . 2 2 )}$ & $\mathbf{0 . 0 1 3}$ \\
\hline
\end{tabular}

Note: Bold indicates data is significant $(p<0.05)$.

\section{Discussion}

Our study showed that clinical response, microbiological response and overall mortality did not differ significantly between the loading dose and non-loading dose groups. However, nephrotoxicity in the loading dose group was significantly higher than in the non-loading dose group.

Previous studies suggested that 9 MIU colistin followed by maintenance dose of 4.5 MIU every 12 hours was an effective and safe therapy for infections due to multidrug-resistant gram negative pathogens. ${ }^{13,14}$ However, the role of a loading dose of colistin has not been definitively established in cancer patients. Previous studies were conducted in only a small number of patients and colistin was administered in general population without immunocompromised status. ${ }^{13,14}$ The clinical response of loading dose colistin for treating XDR-AB infections in our study was lower than previous studies $(53.3 \%$ vs. $63-82.1 \%)^{13,14}$ This might be because of compromised immunity in cancer patients. However, no difference in clinical response between patients receiving loading dose and nonloading dose was found in our study $(53.33 \%$ and $48.15 \%$, respectively; $p=0.661$ ). Microbiological response also was insignificant different between the two groups $(73.33 \%$ and $55.56 \%$, respectively; $p=0.097$ ), although loading dose colistin showed better response. Similarly, a recent retrospective study by Nazer et $\mathrm{al}^{7}$ reported $66.2 \%$ microbiological response in cancer patients infected with carbapenem-resistant $A$. baumannii (CRAB) who were treated with high-dose colistin. ${ }^{7}$

Table 3 Univariate and multivariate logistic regression analysis of independent factors associated with outcomes in cancer patients treated with colistin for XDR-AB $(\mathrm{n}=102)$

\begin{tabular}{|c|c|c|c|c|c|c|}
\hline Outcome and variable & Crude OR & $95 \% \mathrm{Cl}$ & $p$-value & Adjusted OR & $95 \% \mathrm{Cl}$ & $p$-value \\
\hline \multicolumn{7}{|l|}{ Clinical response } \\
\hline Loading dose colistin & 1.23 & $0.5 \mathrm{I}-2.97$ & 0.644 & 1.03 & $0.38-2.75$ & 0.948 \\
\hline Age $\geq 60$ years & 0.77 & $0.35-1.69$ & 0.519 & 1.13 & $0.46-2.80$ & 0.780 \\
\hline Septic shock & 0.30 & $0.13-0.70$ & 0.005 & 0.26 & $0.10-0.64$ & 0.004 \\
\hline Baseline $\mathrm{SCr} \geq \mathrm{I} \mathrm{mg} / \mathrm{dl}$ & 0.61 & $0.26-1.43$ & 0.263 & 0.68 & $0.26-1.77$ & 0.437 \\
\hline Charlson score $\geq 4$ & 0.82 & $0.69-0.98$ & 0.029 & 0.40 & $0.16-0.98$ & 0.047 \\
\hline Duration of colistin $\geq 10$ day & 1.04 & $0.43-2.46$ & 0.929 & 2.83 & $1 .|7-6.8|$ & 0.020 \\
\hline \multicolumn{7}{|l|}{ Microbiological response } \\
\hline Loading dose colistin & 2.2 & $0.88-5.49$ & 0.091 & 2.27 & $0.84-6.13$ & 0.105 \\
\hline Age $\geq 60$ years & 0.81 & $0.35-1.89$ & 0.631 & 1.09 & $0.42-2.80$ & 0.850 \\
\hline Septic shock & 0.31 & $0.12-0.79$ & 0.015 & 0.28 & $0.10-0.78$ & 0.015 \\
\hline Baseline $\mathrm{SCr} \geq \mathrm{I} \mathrm{mg} / \mathrm{dl}$ & 1.00 & $0.4 I-2.48$ & 0.02 & 1.30 & $0.47-3.53$ & 0.606 \\
\hline Charlson score $\geq 4$ & 0.92 & $0.39-2.15$ & 0.844 & 0.93 & $0.37-2.33$ & 0.882 \\
\hline Duration of colistin $\geq 10$ days & 2.22 & $1.00-4.9 \mid$ & 0.048 & 2.49 & $0.98-6.32$ & 0.054 \\
\hline \multicolumn{7}{|l|}{ Nephrotoxicity } \\
\hline Loading dose colistin & 2.59 & $1.05-6.40$ & 0.039 & 5.80 & $1.45-23.25$ & 0.013 \\
\hline Age $\geq 60$ years & 2.05 & $0.89-4.67$ & 0.088 & 3.28 & I.09-9.87 & 0.034 \\
\hline Use vasopressor drug & 1.57 & $0.68-3.63$ & 0.287 & 1.76 & $0.63-4.90$ & 0.278 \\
\hline Baseline $\mathrm{SCr} \geq \mathrm{I} \mathrm{mg} / \mathrm{dl}$ & 0.58 & $0.24-1.39$ & 0.229 & 0.60 & $0.20-1.86$ & 0.385 \\
\hline Duration of colistin $\geq 10$ days & I.II & $0.44-2.75$ & 0.820 & 0.58 & $0.22-1.53$ & 0.274 \\
\hline
\end{tabular}

Abbreviations: $\mathrm{XDR}-\mathrm{AB}$, extensively drug-resistant Acinetobacter baumannii; $\mathrm{OR}$, odds ratio; $\mathrm{Cl}$, confidence interval; $\mathrm{SCr}$, serum creatinine.

Note: Bold indicates data is significant $(p<0.05)$. 
When adjusting the potential confounders by multivariate logistic regression analysis, loading dose colistin was neither associated with clinical response (odds ratio [OR], 1.03; $95 \%$ confidence interval [CI], 0.38-2.75) nor microbiological response (OR, 2.27; 95\% CI, 0.84-6.13) compared with non-loading dose colistin. These results were not significantly different, perhaps because the minimal inhibitory concentration in our study was low (0.064-1.5) in both groups. These results were supported by Álvarez-Marín et $\mathrm{al}^{15}$ who showed that non-loading dose of colistin was effective for treating CRAB ventilator-associated pneumonia caused by strains with high susceptibility to colistin. ${ }^{15}$

Focusing on nephrotoxicity, loading dose colistin significantly increased incidence of nephrotoxicity compared to non-loading ( $50.67 \%$ and $22.22 \%$, respectively; $p=0.013$ ). These results were similar to the study by Nazer et al, ${ }^{7}$ which reported $>60 \%$ nephrotoxicity rate when high-dose colistin $(9$ MIU per day, divided into three doses) was used for treating cancer patients infected with CRAB. ${ }^{7}$

Furthermore, loading dose of colistin was found to be an independent risk factor for nephrotoxicity after adjusting potential confounders (OR, 5.80; 95\% CI, 1.45-23.25) and so high-dose colistin should be used with caution in patients with impaired renal function. Pogue et al ${ }^{16}$ found that nephrotoxicity occurred in patients who received high dose more than those who received low dose of colistin. Their study also showed that nephrotoxicity was dose dependent. The other factor that had correlation with nephrotoxicity in our study was age equal or greater than 60 years (OR, 3.28; 95\% CI, 1.09-9.87).

In addition, a correlation was found between long duration of colistin treatment ( $\geq 10$ days) and clinical response. On the other hand, patients with septic shock or Charlson score $\geq 4$ tended to have poor clinical response. Also, septic shock was a risk factor for poor microbiological response. The study likewise suggested that if critically ill cancer patients suffered from $\mathrm{AB}$ infection, then they would have poor outcomes. ${ }^{17}$

Our study has several limitations. This was a retrospective study and so it might contain inherent bias and incompletion in data collection. Further study therefore should be performed prospectively to confirm these results and determine the optimal dose of colistin for XDR-AB treatment. In addition, our study did not measure serum concentration of colistin because it was not measured routinely in the hospital. Sample size could be considered as a limitation in our study as well. Nevertheless, to the best of our knowledge, our study is the first and largest-to-date to compare loading dose with non-loading dose of colistin in the treatment of cancer patients with XDR-AB-related infections.

In conclusion, loading dose colistin did not affect clinical response, microbiological response and mortality rate in cancer patients with XDR-AB-related infections but significantly increased nephrotoxicity rate. Loading dose colistin therefore should be given carefully in elderly patients or patients with renal impairment and avoided in patients infected with highly colistin-sensitive $A$. baumannii. In addition, if patients with Charlson score $\geq 4$ or septic shock, poor clinical and microbiological responses should be taken into account and properly managed.

\section{Acknowledgment}

We would like to acknowledge Wilai Baosoung for providing the microbiological data.

\section{Disclosure}

The authors report no conflicts of interest in this work.

\section{Reference}

1. Trecarichi EM, Tumbarello M. Antimicrobial-resistant Gram-negative bacteria in febrile neutropenic patients with cancer: current epidemiology and clinical impact. Curr Opin Infect Dis. 2014;27(2):200-210.

2. Montefour K, Frieden J, Hurst S, et al. Acinetobacter baumannii: an emerging multidrug-resistant pathogen in critical care. Crit Care Nurse. 2008;28(1):15-25.

3. Manchanda V, Sanchaita S, Singh N. Multidrug resistant Acinetobacter. J Glob Infect Dis. 2010;2(3):291-304.

4. Chiang MC, Kuo SC, Chen SJ, et al. Clinical characteristics and outcomes of bacteremia due to different genomic species of Acinetobacter baumannii complex in patients with solid tumors. Infection. 2012;40(1):19-26.

5. Turkoglu M, Mirza E, Tunçcan OG, et al. Acinetobacter baumannii infection in patients with hematologic malignancies in intensive care unit: risk factors and impact on mortality. J Crit Care. 2011;26(5): 460-467.

6. Durante-Mangoni E, Signoriello G, Andini R, et al. Colistin and rifampicin compared with colistin alone for the treatment of serious infections due to extensively drug-resistant Acinetobacter baumannii: a multicenter, randomized clinical trial. Clin Infect Dis. 2013;57(3):349-358.

7. Nazer LH, Rihani S, Hawari FI, Le J. High-dose colistin for microbiologically documented serious respiratory infections associated with carbapenem-resistant Acinetobacter baumannii in critically ill cancer patients: a retrospective cohort study. Infect Dis (Lond). 2015;47(11):755-760.

8. Hachem RY, Chemaly RF, Ahmar CA, et al. Colistin is effective in treatment of infections caused by multidrug-resistant Pseudomonas aeruginosa in cancer patients. Antimicrob Agents Chemother. 2007;51(6):1905-1911.

9. Durakovic N, Radojcic V, Boban A, et al. Efficacy and safety of colistin in the treatment of infections caused by multidrug-resistant Pseudomonas aeruginosa in patients with hematologic malignancy: a matched pair analysis. Intern Med. 2011;50(9):1009-1013.

10. Horan TC, Andrus M, Dudeck MA. CDC/NHSN surveillance definition of health care-associated infection and criteria for specific types of infections in the acute care setting. Am J Infect Control. 2008;36(5):309-332. 
11. Clinical and Laboratory Standards Institute. (2007) Performance standards for antimicrobial susceptibility testing-17th Informational supplement. CLSI document M100-S17. CLSI, Wayne, USA.

12. Kellum JA, Bellomo R, Ronco C. Definition and classification of acute kidney injury. Nephron Clin Pract. 2008;109(4):c182-c187.

13. Dalfino L, Puntillo F, Mosca A, et al. High-dose, extended-interval colistin administration in critically ill patients: is this the right dosing strategy? A preliminary study. Clin Infect Dis. 2012;54(12):1720-1726.

14. Trifi A, Abdellatif S, Daly F, et al. Efficacy and toxicity of high-dose colistin in multidrug-resistant gram-negative bacilli infections: a comparative study of a matched series. Chemotherapy. 2016;61(4):190-196.
15. Álvarez-Marín R, López-Rojas R, Márquez JA, et al. Colistin dosage without loading dose is efficacious when treating carbapenem-resistant $\mathrm{Aci}$ netobacter baumannii ventilator-associated pneumonia caused by strains with high susceptibility to colistin. PLoS One. 2016;11(12):e0168468.

16. Pogue JM, Lee J, Marchaim D, et al. Incidence of and risk factors for colistin-associated nephrotoxicity in a large academic health system. Clin Infect Dis. 2011;53(9):879-884.

17. Nazer LH, Kharabsheh A, Rimawi D, Mubarak S, Hawari F. Characteristics and outcomes of Acinetobacter baumannii infections in critically ill patients with cancer: a matched case-control study. Microb Drug Resist. 2015;21(5):556-561.

\section{Publish your work in this journal}

Infection and Drug Resistance is an international, peer-reviewed openaccess journal that focuses on the optimal treatment of infection (bacterial, fungal and viral) and the development and institution of preventive strategies to minimize the development and spread of resistance. The journal is specifically concerned with the epidemiology of antibiotic resistance and the mechanisms of resistance development and diffusion in both hospitals and the community. The manuscript management system is completely online and includes a very quick and fair peerreview system, which is all easy to use. Visit http://www.dovepress.com/ testimonials.php to read real quotes from published authors. 